\title{
ESTRATEGIAS DE APROPIACIÓN Y APUESTAS DEL DESARROLLO SOSTENIBLE POR LAS COMUNIDADES NEGRAS E INDÍGENAS EMBERA DEL PACÍFICO NORTE COLOMBIANO: EL CASO DE LA CARRETERA ÁNIMAS - NUQUÍ, CHOCÓ (COLOMBIA)
}

\author{
Gonzalo Cocomá Arciniegas ${ }^{62}$
}

\begin{abstract}
RESUMEN
Este artículo explora las recomposiciones territoriales y sociales asociadas con proyectos de desarrollo en áreas rurales. El caso involucra a la población negra e indígena Embera del Departamento del Chocó en el Pacífico Norte colombiano y a los actores relacionados con la construcción de una carretera hacia el Océano Pacífico. La pregunta que dirige esta investigación es: ¿cuáles son los cambios provocados por un proyecto de desarrollo planeado por el Estado en territorios de propiedad colectiva de minorías étnicas? En efecto, la carretera cruza territorios colectivos de comunidades negras e indígenas y a partir de esta problemática, nos preguntamos por los actores implicados, por sus apuestas y sus estrategias de acción. De la misma manera, considerando que el proyecto de la carretera es portador de una lógica de desarrollo, que se desea sostenible, nos preguntamos por las estrategias de las comunidades para apropiarse de la noción de desarrollo sostenible.
\end{abstract}

Palabras clave: proyectos de desarrollo, desarrollo sostenible, comunidades (negras e indígenas), apropiación.

\begin{abstract}
The article explores the territorial and social changes linked to development projects in rural areas. The case involves the black population and indigenous Embera of Chocó (a department in the northwest Pacific Coast of Colombia) as well as other social actors affected by the construction of a road to the Pacific Ocean. The question that directs this investigation is: which are the changes provoked by a project of development planned by the State in territories of collective property of ethnic minorities? In effect this route will cross these collectively held territories, which leads to questions regarding the social actors, their stakes in this project and the strategies they have deployed. Along the same lines and considering that this project is marketed as a strategy for sustainable development, it was necessary to understand how this concept was being appropriated and reinterpreted by local communities.
\end{abstract}

Key words: development projects, collectively held territories, sustainable development, communities.

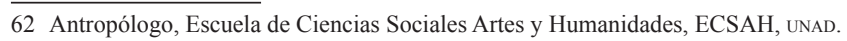




\section{INTRODUCCIÓN}

El tema de esta investigación es las relaciones entre modernidad y desarrollo, específicamente, las estrategias de apropiación del desarrollo sostenible por la población afrocolombiana rural y las comunidades indígenas Embera del Departamento del Chocó en la Costa Pacífica colombiana, que involucra también a otros actores e instituciones que intervienen en el caso de estudio: la construcción de la carretera entre las poblaciones Ánimas - Nuquí del mismo Departamento.

El trabajo analiza los procesos de construcción de Estado-Nación en Colombia y las dinámicas de recomposición territorial y social de poblaciones locales, asociadas a proyectos de desarrollo. El hecho puntual, la continuación de la carretera al mar en el Departamento del Chocó entre los pueblos las Ánimas y Nuquí, hace parte de este proceso de construcción del Estado moderno en un territorio que ha sido histórica y socialmente marginado. La culminación de este proyecto ha mantenido expectante a la población chocoana por más de 50 años, generando polémicas, debates y concertaciones entre los actores implicados, que desde sus propias perspectivas e intereses interactúan con otros actores sociales (Crozier y Friedberg, 1977), haciendo de este problema una de las negociaciones más álgidas de la región en los últimos años (Díaz, 2005).

La pregunta guía de este trabajo es: ¿cuáles son los cambios (culturales, sociales, políticos, medioambientales y económicos) asociados con este proyecto en los territorios de propiedad colectiva (aunque la propiedad es colectiva, el patrón de asentamiento de estos grupos es ribereño, rural y disperso) de comunidades afrodescendientes e indígenas Embera, en el Pacífico Norte colombiano, Departamento del Chocó?

Teniendo en cuenta que este proyecto obedece a una lógica de desarrollo que se desea sostenible, nuestros interrogantes giran en torno a las estrategias de apropiación de dicho desarrollo por parte de las comunidades negras e indígenas, a la manera como se va filtrando el desarrollo sostenible en la memoria regional, al imaginario de modernidad que tienen estos grupos sociales, a la institucionalización del desarrollo en las poblaciones locales, al impacto que tiene sobre la conservación de la biodiversidad y a las respuestas que estos grupos dan a la imagen que tienen de "atrasados" o "tradicionales" (PEDROSA 1996: 68).

\section{METODOLOGÍA}

Como marco metodológico para el análisis del problema hemos empleado tres aproximaciones que exploran las relaciones entre actores sociales diversos y un conjunto social mayor en el cual se confrontan: la Sociología de la traducción, la Socio-antropología del desarrollo y la teoría del actor y el sistema. 


\section{La sociología de la traducción}

Analiza las relaciones de poder inherentes a toda empresa, considerada esta como una organización, campo de enfrentamientos, de cooperaciones y sobre todo un espacio de relaciones donde los actores intentan maximizar sus ganancias, o al menos no dilapidar sus recursos. Para los sociólogos Bruno Latour y Michel Callon (citados por Amblard et al., 1996), principales exponentes de esta tradición sociológica, la regulación de las organizaciones pasa por la búsqueda de un equilibrio entre los deseos y las estrategias expresadas por los actores. Este es un equilibrio frágil donde se pone en juego una serie de recursos humanos, razón por la cual están presentes las relaciones de fuerza o de poder. La sociología de la traducción analiza entonces la emergencia de hechos científicos y sociales en sus contextos particulares, preguntándose por las condiciones en las cuales los actores de una configuración específica convergen alrededor de un cambio social o de una innovación.

\section{Socio-antropología del desarrollo}

La socio-antropología es el estudio empírico de los grupos sociales contemporáneos y de sus interacciones, en una perspectiva diacrónica. De acuerdo con esto, el desarrollo es un campo privilegiado en el que convergen múltiples actores sociales heterogéneos, convirtiéndose en otra forma de cambio social, que no es exclusivo de las sociedades occidentales. En esta perspectiva, el desarrollo no es un ideal ni una calamidad, sino un objeto de estudio del cual se puede dar cuenta mediante la investigación y el análisis empírico que permita describir y analizar las prácticas, representaciones y acciones que le son asociadas (Olivier de Sardan, 1998). La socio-antropología aporta una dimensión comprensiva del desarrollo, gracias al análisis de las asimetrías entre los actores, los diversos intereses que los motivan y sus propias racionalidades. Resaltamos que la cotidianidad del desarrollo está compuesta más bien por compromisos, interacciones y negociaciones que por proyectos y programas específicos y es alrededor de estas nociones que este se debe entender. La dinámica del desarrollo hecha de negociaciones es comparable a una "arena" (Bierschenk 2000: 7), donde los actores confrontan sus diferencias, sus estrategias e intereses con otros actores.

\section{Teoría del actor y el sistema}

Surge del análisis sociológico de las organizaciones, consideradas éstas como conjuntos complejos e integrados, como de los medios e instrumentos inventados y utilizados por los seres humanos para asegurar la cooperación en vista de objetivos comunes. Para Michel Crozier y Erhard Friedberg (1977), la problemática se encuentra alrededor de la acción colectiva de los seres humanos, así como sobre las condiciones y dificultades que lo hacen posible. El análisis se centra en la relación entre actores sociales y sistemas, ya que los actores no existen por fuera de este, el cual define su libertad y la racionalidad de su acción, y a su vez es el actor el único que porta el sistema, le da sentido y lo cambia. Esta relación actor sistema se construye con base en la reciprocidad y la dependencia mutua. Se parte de la idea que la acción colectiva no es un fenómeno natural, sino una construcción social específica que los actores (más o menos autónomos, con acceso a recursos y con sus particularidades) 
han creado, inventado o instituido para resolver los problemas, especialmente aquellos que implican objetivos comunes. Partiendo de la idea que toda acción colectiva es producto, efecto y hecho del poder, esta se constituye como un sistema del mismo. Es el poder el que se ordena y regulariza la acción colectiva permitiendo que los seres humanos puedan cooperar mutuamente en empresas colectivas.

Estas tres corrientes sociológicas son complementarias para el análisis del problema de investigación, el cual identificamos claramente como una interacción compleja de personas y de instituciones alrededor de un objetivo común: la construcción de una carretera.

Esta presentación hace parte de un proceso de investigación doctoral, la cual presenta los elementos que componen la problemática y la problemática en sí misma. A modo de conclusión se presenta una discusión de una de nuestras preguntas de trabajo, a partir de datos recolectados en entrevistas, trabajos de campo realizados y revisión bibliográfica.

\section{CONTEXTO DE LA PROBLEMÁTICA}

Es necesario tener en cuenta ciertos aspectos, que aunque se presenten separados están estrechamente relacionados y deben considerarse como un conjunto para la comprensión del problema. El primero de ellos corresponde a las características geográficas y medioambientales de la región, las cuales hacen de esta, una zona importante para la conservación y protección de la biodiversidad (Ruíz et al., 1993). Las características geográficas de esta región la convierten en un área de gran diversidad biológica: localizada en la franja tropical de América del Sur y azotada por los vientos provenientes del Océano Pacífico que chocan contra la barrera natural de los Andes, esta región es una de las más húmedas del planeta (Eslava, 1993, pp. 138-139). Esta combinación de calor y humedad es propicia para la formación de ecosistemas óptimos que favorecen la presencia de diversas especies endémicas de animales y plantas (Alberico 1993: 236; Stiles 1993: 249-250; Rangel y Lowy 1993: 196-197; Gentry 1993: 202).

El segundo aspecto es el elemento humano, su historia y los aspectos socioculturales. En esta región se encontraban los grupos indígenas Embera, Waunana, Cuna y Tule (Vargas, 1993: 294). Luego, la región fue conquistada y colonizada a finales del siglo XVII por los españoles, los cuales nunca buscaron establecerse allí sino enriquecerse con la explotación de oro (Werner, 2000: 11-12). Por último, fue una de las zonas a las que fue traída población africana esclavizada para reemplazar la mano de obra indígena ya diezmados en la conquista, para trabajar en la minería principalmente así como en la agricultura y servicios domésticos (Colmenares, 1978: 276). Estos tres grupos humanos conforman el aspecto humano del problema, dentro del cual resaltamos los diferentes procesos de resistencia por parte de indios y negros (Werner Cantor op.cit: 129; Sharp 1993: 406), el proceso de mestizaje entre estos tres grupos humanos (Colmenares op.cit: 410), y los aspectos sociales y culturales afectados por la construcción de la carretera (formas tradicionales de producción, saberes locales, organización social, prácticas mágico - religiosas, fiestas y tradiciones populares, etc.). 
El tercer aspecto es la política. Este elemento, que ha incidido mucho en el aspecto económico, debe comprenderse a partir de una perspectiva cronológica que abarque desde la colonia hasta nuestros días. De esta manera es posible entender los cambios que han tenido las leyes que tratan sobre población indígena y negra en el país (Mosquera, 2004: 7). La actual Constitución Colombiana de 1991, que reemplazó la antigua constitución de 1886, que se mantuvo vigente por más de cien años con sus reformas pero nunca con una modificación profunda, significó un cambio radical respecto a su predecesora. Se reconoció por primera vez en la historia del país la multiétnicidad y la pluriculturalidad de la nación: negros e indígenas fueron reconocidos como ciudadanos y pilares de la cultura y de la identidad nacional. En el caso de las comunidades negras se aprobó el artículo transitorio 55 mediante el cual el Estado colombiano se comprometió a crear una ley que "reconozca a las comunidades negras que han venido ocupando tierras baldías en las zonas rurales ribereñas de los ríos de la Cuenca del Pacífico, de acuerdo con sus prácticas tradicionales de producción, el derecho a la propiedad colectiva sobre las áreas que habrá de demarcar la misma ley" (Constitución Nacional de Colombia: artículo transitorio n. ${ }^{\circ}$ 55). Esta ley es la Ley 70 de Agosto 27 de 1993, también conocida como Ley de Negritudes, la cual reconoce la propiedad colectiva a las comunidades negras de la Cuenca del Pacífico los territorios baldíos que han venido ocupando y explotando ${ }^{63}$ con sus prácticas tradicionales de producción.

El cuarto aspecto es el contexto económico que, como ya dijimos, está estrechamente relacionado con el aspecto político. Históricamente, el Pacífico ha sido considerado como una región a colonizar de la cual se deben aprovechar sus recursos naturales. ${ }^{64}$ Desde La Colonia hasta nuestros días han sido diversos los periodos de extracción de productos de la selva, relacionados con auges económicos promocionados desde el gobierno central (tagua, caucho, maderas). Estos periodos de auge de explotación económica, caracterizados por la extracción de materias primas, han estado vinculados a políticas de apropiación del territorio y presencia estatal en la región, siendo el último de ellos los proyectos agroindustriales: banano, palma de aceite, camaroneras; y los megaproyectos de desarrollo: construcción de carreteras, infraestructura, puertos (Escobar, 1996: 124).

Estos cuatro aspectos conforman el contexto del problema de investigación, dentro del cual hace falta mencionar a los actores involucrados en el mismo. Estos actores son: las comunidades afrocolombianas e indígenas Embera, las instituciones del Estado (Instituto Nacional de Vías -INVIAS-, el Ministerio del Medio Ambiente), la Universidad Tecnológica del Chocó Sergio Luis Córdoba -UTCH-, y la población urbana y rural del Chocó que no está en el área de influencia de la carretera. Estos son los actores que están directamente

63 Esta ley presenta una serie de fallas: se aplica únicamente a las comunidades negras de la cuenca del Pacífico, como si no existieran otras comunidades negras en el resto del país; los trámites necesarios para legalizar los territorios de las comunidades negras son complejos y motivo de conflictos en las comunidades; se reconoce como comunidad negra solamente a la población rural excluyéndose a la población urbana (ver: AGIER, M y HOFFMANN, O., 1999. Les terres des communautés noires dans le pacifique colombien. Interprétations de la loi et stratégies d'acteurs. En: problèmes d'Amérique latine, n. ${ }^{\circ} 32$, jan-mars 1999, pp. $17-42$.

64 Durante el siglo XVIII la explotación aurífera del Chocó alcanzó sus máximos niveles de productividad, siendo el virreinato de la Nueva Granada (actual Colombia) la que mayor cantidad de oro aportó a la corona española (WERNER CANTOR Op.cit: 19). 
relacionados con la carretera, sin embargo, se debe considerar la incidencia de otros grupos en la realización de este proyecto: grupos armados, los grupos económicos regionales (del Eje cafetero especialmente) y el resto de la sociedad chocoana y colombiana. Estos grupos de actores heterogéneos, involucrados a su manera en el proceso de construcción de la carretera, tienen sus apuestas individuales y colectivas que se ponen en juego con las apuestas de los otros actores, formando una especie de red o entramado de relaciones sociales de diferentes tipos que se transforman a medida que el proceso avanza. Estas relaciones son dinámicas, cambiantes de acuerdo con el contexto específico y a la posición que asuman los actores en

un momento dado, en concordancia con las estrategias empleadas por ellos mismos y los demás actores (Bernoux 1985: 124).

\section{Problemática: la carretera al mar, el desarrollo sostenible y las comunidades negras e indígenas embera del chocó}

La construcción de la vía al mar, como se conoce este proyecto, ha estado ligada al deseo nacional y regional de tener una salida en la Costa Norte colombiana sobre el Océano Pacífico así como de conexión intercontinental entre el sur, el centro y el norte de América. Este proyecto surge como parte del interés nacional en ampliar sus horizontes económicos y exploración de la Cuenca del Pacífico (Pedrosa 1996: 71). Sus antecedentes históricos se remontan a la primera mitad del siglo XX, cuando se construyó un tramo de la carretera sin estudios ambientales ni concertaciones con las comunidades locales, lo cual no tuvo buena aceptación por parte de las personas de la zona. Durante un tiempo se percibió como un elemento exógeno a la lógica tradicional local y como una falta de respeto a las comunidades locales (especialmente a comunidades indígenas Embera) ya que el proyecto atraviesa sus territorios y éstas no fueron consultadas. Además, la carretera representa también una serie de amenazas para las culturas locales, como por ejemplo la llegada de personas extrañas que no son de la región: "paisas (gente proveniente de Antioqui el viejo Caldas)", "rolos (gente de la Sabana de Bogotá)" y de otras partes del país ${ }^{65}$ así como la incursión de empresas privadas y particulares que quieren comprar sus territorios. Sin embargo, el proyecto de la vía al mar, que ha provocado un cambio en la representación del territorio por parte de los habitantes locales, es actualmente una esperanza para la unificación del pueblo chocoano (Salgado 2005: comunicación personal).

Originalmente, la carretera debía unir a la capital chocoana, Quibdó, con el municipio de Bahía Solano, como parte del plan nacional de ocupación y utilización de territorios baldíos y la creación de colonias agrícolas con campesinos del interior del país, y de allí debía continuar hacia Panamá, luego de que se terminara la carretera que unía Medellín con Quibdó. Esta última fue terminada en la década de los años cuarenta. Tres décadas pasaron y la carretera sólo había llegado hasta Las Ánimas, a escasos 22 de kilómetros al sur de Quibdó: paros y protestas civiles, falta de presupuesto, la geografía de la región, hicieron que la historia de este haya sido interrumpido en diversas ocasiones (Díaz, 2005).

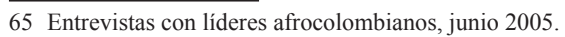




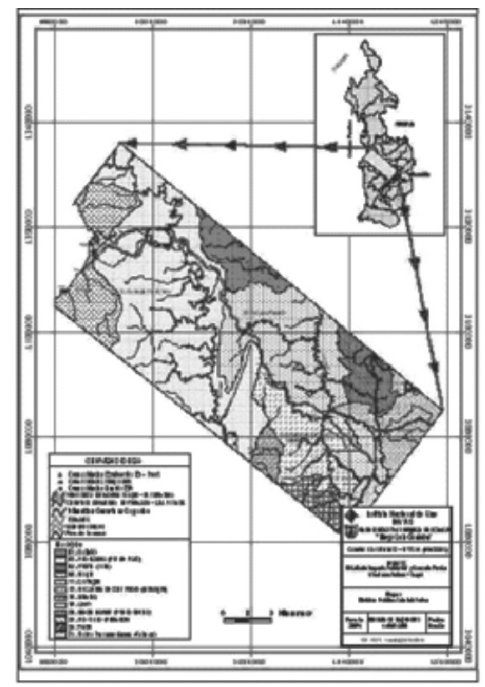

Ilustración 1. Zona de influencia de la carretera en el Chocó, fuente: http://www.beteguma.org/antecedentesvia.htm

La continuación de esta carretera representa la unificación del territorio chocoano y de sus gentes. Ejemplo de ello es que los habitantes del litoral se refieren a los habitantes del interior del Departamento como "chocoanos" y muchos de estos últimos no conocen siquiera el Océano. Sin embargo, este proceso es reconocido entre la población más como un debate y una negociación intensa entre los actores implicados, que como la realización de acciones concretas por parte del Estado. Es contradictorio, según líderes afrocolombianos, que siendo este un proyecto impulsado por el Estado, sea el mismo Estado el que le pone obstáculos con su estructura institucional, burocrática y centralista. ${ }^{66}$ En efecto, las discusiones alrededor de la carretera al mar ha sido un tema candente que ha permeado diferentes esferas tanto a nivel local como nacional durante varios años y que ha dividido opiniones al respecto: en este caso las entidades responsables de la construcción de carreteras y el ministerio del medio ambiente.

Estas opiniones divididas que existen respecto a la construcción de la carretera aparecen en un reciente trabajo de campo realizado en Quibdó (junio 2006) y en entrevistas hechas en Bogotá: existen por lo menos tres percepciones o visiones del proyecto. La primera es la visón de los actores de la capital del país, quienes se inclinan en su mayoría a defender una posición ambientalista de conservación y protección de la selva chocoana argumentando la fragilidad del ecosistema y lo biológico como hecho social global. Una segunda percepción es la que se tiene en la región, representada especialmente por los habitantes de la capital chocoana, Quibdó, quienes manifiestan el deseo de tener la carretera a cualquier costo, pero se percibe cierto escepticismo sobre su realización. Por último, está la percepción de las

66 Entrevistas: comunicación personal con Juan de Dios Mosquera, director del movimiento nacional por los DDHH de las comunidades negras (Bogotá, abril 2006) y con Idalmis Minota, acompañante del proceso del consejo comunitario de San Francisco de Cugucho en el alto Baudó (Quibdó, junio 2006). 
comunidades indígenas y afrocolombianas locales de la zona de influencia de la carretera. Estas comunidades se pueden dividir en dos grupos: las comunidades por las cuales ya se ha construido la carretera y aquellas donde se irá a construir. Quienes ya cuentan la carretera en sus territorios han visto cómo esta ha sido descuidada y evalúan después de este tiempo las consecuencias que esta ha traído: cambio en el patrón de asentamiento (del río a la carretera), cambios en las formas de producción, abandono de actividades tradicionales, monetarización, entre otros. Las otras comunidades en las cuales no se ha construido, ven en ella tantas oportunidades como amenazas y aunque desean que la carretera se haga, no quieren que se repita la experiencia del tramo ya construido: que no se les consulte o no se les den garantías para proteger el bosque y sus culturas. ${ }^{67}$

Teniendo en cuenta que en la región se han llevado a cabo procesos de titulación de territorios colectivos a nombre de las comunidades negras desde 1994, y que estos trámites son complejos y a su vez generadores de conflictos, la concertación para la continuación de dicha carretera en el tramo Animas - Nuquí, se suma como otra fuente de conflictos entre los actores sociales mencionados arriba.

Otro aspecto del problema que debe considerarse, son las condiciones bajo las cuales es planteado el proyecto: este se concibe bajo los parámetros de desarrollo sostenible, noción que se confronta con las ideas propias que tienen las comunidades sobre el territorio, sobre sus prácticas productivas y su visión de futuro. Además, por realizarse este proyecto en un área de alta biodiversidad, los actores implicados ponen en juego la protección y conservación del medio ambiente con sus acciones y estrategias.

\section{Breve recuento de la carretera Ánimas-Nuquí}

La infraestructura vial del Chocó es casi inexistente, en mal estado y se ha hecho por pedazos: en 1966 el Departamento contaba con $230 \mathrm{Km}$ de carreteras nacionales, el año pasado se contabilizaron $300 \mathrm{~km}$. Los estudios para el trazado de la vía al mar se iniciaron en la década del 40 del pasado siglo y en esa misma década se iniciaron los primeros trabajos de construcción, sin embargo, los trabajos se interrumpieron en 1964 por diversos problemas de orden político, técnicos, ecológicos y socioeconómicos. La construcción se reanudó en 1967 construyéndose 14 kilómetros hasta el río Mungidó. En 1967 una empresa privada avanza del Km. 14 al 22. En 1970 se adjudicó la construcción de un puente sobre el río San Pablo a una firma y la continuación de la vía a la misma empresa que había ejecutado los trabajos en 1967, que de manera intermitente y discontinua abrió la vía hasta el Km. 70. Los trabajos se interrumpieron en agosto de 1992 cuando dirigentes indígenas bloquearon la vía, exigiendo respeto por las culturas locales, que se hicieran estudios ambientales y que se tuviera en cuenta su opinión sobre la construcción de la carretera (Díaz, 2005).

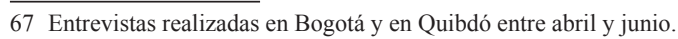


En el año 2000, luego de un paro cívico de la población chocoana, el gobierno nacional se comprometió a terminarla. En el año 2005, el debate ha vuelto a ser de actualidad: el Instituto Nacional de Vías (INVIAS), organismo encargado de construir, mantener y consolidar el sistema de infraestructura de transporte en Colombia, firmó un convenio con la Universidad Tecnológica del Chocó, Sergio Luis Córdoba (UTCH), para que realice los trabajos necesarios (Consulta Previa con las comunidades negras e indígenas y el Estudio de Impacto Ambiental) para obtener la licencia ambiental del proyecto y poder terminarla. Parte de los fracasos anteriores por terminar la carretera era precisamente la falta de concertación con las comunidades locales y la ausencia de estudios ambientales sobre los impactos de la misma. En las últimas semanas la UTCH entregó el documento final a INVIAS para su evaluación y aprobación, decisión última que debe ser tomada por el ministerio del medio ambiente. Actualmente la CP y el EIA están en manos del Ministerio del Medio Ambiente, de quien se espera emita la licencia ambiental para la finalización del proyecto.

\section{A MODO DE CONCLUSIÓN}

La continuación de la vía al mar en territorios de comunidades afrocolombianas e indígenas Embera en el Departamento del Chocó, es otra fuente de "conflictos" territoriales entre estos grupos sociales, instituciones del Estado y el resto de la población chocoana, del Eje cafetero y nacional. La construcción de la carretera se percibe como un proyecto exógeno, que surge de los intereses del poder central del Estado y de las regiones económicamente más fuertes que el Chocó. Este proyecto moviliza una serie de ideas (desarrollo sostenible, modernidad, progreso) que se confrontan con las ideas propias que tienen las comunidades sobre su territorio, su visión de futuro, sus formas tradicionales de producción y su cultura. Asimismo, pone en juego la protección y conservación del medio ambiente en la región bajo las consignas del desarrollo sostenible, por todos los actores involucrados en el mismo.

Por tanto, la pregunta que nos hacemos hace parte de las preocupaciones de las ciencias sociales y la relación desarrollo - modernidad: Pedrosa (1996), se cuestiona sobre la apropiación del discurso del desarrollo sostenible a partir del análisis de las formas de infiltración del discurso y de las respuestas que dan las comunidades a ello. Por su parte Olivier (1998), se pregunta por el vínculo entre el lenguaje del desarrollo (lenguaje técnicocientífico) y los saberes locales, centrándose en el papel que cumplen los intermediarios en esta relación.

En tanto que discurso y práctica representativa de un hecho ligado a cambios sociales en poblaciones rurales, el desarrollo sostenible es una noción todavía floja en las representaciones de las comunidades que lo utilizan, pero que comienza a ser integrado en la retórica local gracias a los procesos mediación y a las personas que lo efectúan (courtier en francés y brokers en inglés). Este proceso tiene lugar en las arenas (locales, regionales, nacionales o internacionales) donde se encuentran reunidos diferentes actores o grupos estratégicos, movidos por intereses y objetivos comunes, ligados en una red de intercambios de saberes, técnicas y conocimientos. Sin embargo, estos intercambios no son necesariamente materiales, pueden y de hecho son también simbólicos. Por lo tanto, el mecanismo de apropiación de este 
discurso se hace esencialmente con palabras, que sirven como herramientas metodológicas en un trabajo de campo.

Para finalizar esta presentación, discutiremos una de nuestras preguntas de trabajo de investigación. Si pensamos que efectivamente existe una apropiación del desarrollo sostenible por parte de las comunidades afrocolombianas e indígenas del Pacífico norte colombiano, nos preguntamos entonces por los factores que promueven esta apropiación. Nuestra pregunta se dirige entonces a escudriñar las razones por las cuales esta apropiación se convierte en una estrategia por parte de estas comunidades.

En las entrevistas realizadas hasta el momento, nos hemos dado cuenta de que el uso del desarrollo sostenible no es general a toda la población de comunidades negras e indígenas. Este es empleado por los líderes y representantes de las comunidades, especialmente ante funcionarios del Estado, investigadores, miembros de $\mathrm{ONG}$, entre otros.

Otro aspecto que vale la pena resaltar es la visión que tienen estos líderes locales del propio desarrollo sostenible. La manera como se refieren a él, no proviene del estudio o de una reflexión propia por parte de los líderes o de las personas de las comunidades, sino que ha sido introducido por los actores exógenos al contexto local (antropólogos, miembros de ONG, investigadores, agentes del Estado, etc.), pero que adquiere una connotación diferente en los líderes y representantes de comunidades. Ellos, por estar en una posición de "negociadores" y de "representantes" de un grupo social que tienen relaciones particulares con el medio que los rodea, manifiestan que el "desarrollo sostenible" no ha sido una novedad para ellos ya que todas sus prácticas de producción han sido "sostenibles" durante años.

En contraste con esta visión "conservacionista" de los propios líderes afrocolombianos e indígenas, hay opiniones de otros líderes de estas mismas comunidades que están en desacuerdo con esta versión (Entrevista Juan de Dios Mosquera, Bogotá, abril de 2006, comunicación personal). Para ellos las prácticas productivas tradicionales, que han sido consideradas como prácticas productivas sostenibles, no son otra cosa que estrategias de supervivencia de las comunidades locales (negras e indígenas) en un medio ambiente que exige una adaptación particular. Lo de la sostenibilidad, continúa, es un rótulo que se le ha puesto a las actividades económicas de los grupos que habitan en la selva, por parte de las entidades del Estado. Pero, ¿para qué? se preguntan. El desarrollo sostenible es una forma de excusa de los países desarrollados para guardar y preservar lo que queda de bosques originales o pocos intervenidos, donde habitan comunidades que en el pasado reciente han sido marginadas por el poder central del Estado. Esta es una visión del desarrollo sostenible que se escucha sobre todo en los líderes que dirigen movimientos nacionales de reivindicación de derechos humanos de las comunidades. En esta visión, las comunidades locales son vistas más que todo como víctimas de un engaño por parte de los líderes locales que emplean el mismo lenguaje tecnócrata utilizado por los agentes del Estado, miembros de ONG e investigadores, para sus propios intereses. 
"Desarrollo sostenible" como tal se convierte entonces en una herramienta, muchas veces exclusivamente retórica, para beneficio de un pequeño sector de las comunidades. Con esta herramienta se pretende captar la atención de entidades del Estado y ONG nacionales e internacionales para obtener recursos para sus comunidades. Sin embargo, son los líderes locales quienes manejarán estos dineros, pues fueron ellos quienes lograron captar la atención de las agencias financiadoras y quienes representan a las comunidades, por tanto gozan del privilegio, el prestigio y el beneplácito de la comunidad para ser los administradores locales de los proyectos.

En este sentido, ciertos miembros de las comunidades han desarrollado estrategias definidas y condicionadas por estos elementos: su situación frente a las instituciones de desarrollo, su grado de inserción en las diferentes arenas o redes de negociación de proyectos y la consolidación de su prestigio en estas arenas o redes, tanto locales como nacionales e internacionales.

A partir del comportamiento de estos líderes locales, pensamos que la capacidad de negociar intereses y deseos con las agencias financiadoras de proyectos de desarrollo, reside en el dominio que tengan en lenguaje técnico del desarrollo. Este dominio del lenguaje del desarrollo resulta entonces condición necesaria para la participación en las negociaciones y en la defensa de los intereses colectivos de las comunidades.

Es necesario entonces analizar la intencionalidad de las comunidades. Si esta dinámica del desarrollo ha permitido la apropiación de una "renta" proveniente de las instituciones que financian proyectos, entonces el dominio del lenguaje del desarrollo sostenible le permite a las comunidades afrocolombianas e indígenas captar recursos de la ayuda internacional, a través de la figura del mediador local.

Es claro que las condiciones en las que viven estas comunidades corresponden a un aislamiento no sólo geográfico sino político, económico y social. Ante ello es normal que las comunidades que tienen los más bajos niveles de calidad de vida en el país, deseen tener todo de lo que han sido privadas durante al menos tres siglos. En suma, ha sido la "invisibilización" por parte del Estado lo que ha obligado a estas comunidades a tomar decisiones radicales, como impedir la continuación de la carretera en 1992, para que sean escuchadas sus necesidades, al tiempo que asumen estrategias de riesgo para sí mismas.

Tal es el caso de la figura del mediador, es decir, la elección de una persona que representará y negociará a nombre de las comunidades los intereses colectivos, so pena de proveerlo de un poder que lo pondrá en una posición de desequilibrio ante sus compañeros de colectividad. Sin embargo, es el precio que las comunidades están dispuestas a pagar para tener al menos la oportunidad de ser escuchadas sus peticiones y tener, después de mucho tiempo, la atención del Estado para ser incluidos en la dinámica económica y social de un país que está al otro lado de la cordillera. 


\section{REFERENCIAS BIBLIOGRÁFICAS}

ALBERICO, M., (1993), La zoografía terrestre, págs. 232-239. En: LEYVA, P., 1993 (editor) Colombia Pacífico Tomo I. Proyecto Editorial del Fondo FEN - Colombia, Bogotá. 399 pp.

AMBLARD, H.; P. BERNOUX y G.HERREROS, 1997 (2. ${ }^{\text {a }}$ ed.). Une sociologie de la traduction, pp. 127-180. En : AMBLARD, H.; P. BERNOUX y G.HERREROS (et al.) Les nouvelles approches sociologues des organisations. Editorial du Seuil, Paris, 244 pp.

BERNOUX, P., (1985), La sociologie des organisations. Éditions du Seuil, Paris, 390 pp.

BIERSCHENK, T., J.P. CHAVEAU y J.P OLIVIER de SARDAN. 2000 (coord.) Courtier en developpement: les villages africains en quête de projets. Editorial KARTHALA APAD, Paris, 328 pp.

COLMENARES, G., (1973), Historia económica y social de Colombia 1537-1719. Editorial

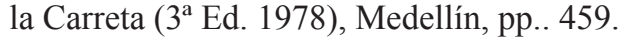

\section{CONSTITUCIÓN NACIONAL DE COLOMBIA DE 1991.}

CROZIER, M. y E. FRIEDBERG, (1977), L'acteur et le système. Editorial de Seuil, Paris, 504 pp.

DÍAZ CAÑADA, G., (2005), Antecedentes históricos de la vía al mar Ánimas-Nuquí: pasado y presente. En: http://www.ibcperu.org/doc/isis/12607.pdf.

ESCOBAR, A. 1996. Viejas y nuevas formas de capital y los dilemas de la biodiversidad, págs. 109-131. En: ESCOBAR, A y PEDROSA, A. (investigadores), 1996. Pacífico ¿desarrollo o diversidad? estado, capital y movimientos sociales en el Pacífico colombiano. Editorial CEREC - ECOFONDO, Santafé de Bogotá, 373 págs.

ESLAVA, J.A., (1993), Climatología, págs. 136-147. En : LEYVA, P., 1993 (editor) Colombia Pacífico Tomo I. Proyecto Editorial del Fondo FEN - Colombia, Bogotá. 399 pp.

GAMBOA, J., (1999), La política de baldíos en el Chocó durante la segunda mitad del siglo XIX, págs. 127-158. En : VARGAS SARMIENTO, P., 1999 (coor. y comp.). Construcción territorial en el Chocó. Volumen I: historias regionales. ICAN-PNR-OBAPO, Bogotá, 202 pp.

GENTRY, A.H., (1993), Riqueza de especies y composición florística, págs. 200-219. En : LEYVA, P., 1993 (editor) Colombia Pacífico Tomo I. Proyecto Editorial del Fondo FEN - Colombia, Bogotá. pp. 
MOSQUERA, S., (2004), La gente negra en la legislación colonial. Editorial Lealon - serie Ma'Mawu, Medellín, 188 pp.

OLIVIER DE SARDAN, O., (1998), Anthropologie et développement. Editorial : APAD Karthala, Paris, 224 pp.

PEDROSA, A., (1996), La institucionalización del desarrollo, págs. 66-89. En : ESCOBAR, A y PEDROSA, A. (investigadores), 1996. Pacífico ¿desarrollo o diversidad?: estado, capital y movimientos sociales en el Pacífico colombiano. Editorial CEREC - ECOFONDO, Santafé de Bogotá, 373 pp.

RANGEL, J.O. y P. LOWY, (1993), Tipos de vegetación y rasgos fitográficos, págs. 182-198. En : LEYVA, P., 1993 (editor) Colombia Pacífico Tomo I. Proyecto Editorial del Fondo FEN - Colombia, Bogotá, 399 pp.

RIST, G., (2001), (2.e édiction) Le développement : histoire d'une croyance occidentale. Editorial : Press de sciences Po, Paris, 443 pp.

SALGADO, Jorge Iván periodista del semanario Chocó 7 días. Entrevista: viernes 23 de Junio 2006. Quibdó.

SHARP, W.F., (1993), Manumisión, libres y resistencia negra en el Chocó colombiano, págs. 406-419. En : LEYVA, P., 1993 (editor) Colombia Pacífico Tomo II. Proyecto Editorial del Fondo FEN - Colombia, Bogotá. 474 pp.

STILES, F.G., (1993), La avifauna, págs. 248-255. En : LEYVA, P., 1993 (editor) Colombia Pacífico Tomo I. Proyecto Editorial del Fondo FEN - Colombia, Bogotá. 399 pp.

VARGAS SARMIENTO, P., (1993), Los embera, Waunana y los Cuna : cinco siglos de transformaciones territoriales en la región del Chocó, págs. 292-309. En : LEYVA, P., 1993 (editor) Colombia Pacífico Tomo I. Proyecto Editorial del Fondo FEN - Colombia, Bogotá, 399 pp.

WERNER CANTOR, E., (2000), Ni aniquilados, ni vencidos : los Emberá y la gente negra del Atrato bajo el dominio español. Siglo XVIII. Editorial : Instituto Colombiano de Antropología e Historia, Bogotá, 202 pp. 